\title{
Expression profile of IncRNAs and mRNAs in intestinal macrophages
}

\author{
QAIS AHMAD NASEER ${ }^{1,2^{*}}$, LULU LIU $^{1 *}$, XIAOFEI XUE ${ }^{3 *}$, SICHE CHEN $^{1 *}$, JIXIANG CHEN $^{1}$, \\ JIANGUO QU ${ }^{1}$, LEI CUI ${ }^{1}$, XIAOGANG WANG ${ }^{3}$ and SHENGCHUN DANG ${ }^{1,3}$ \\ ${ }^{1}$ Department of General Surgery, The Affiliated Hospital, Jiangsu University, Zhenjiang, Jiangsu 212001; ${ }^{2}$ Department of \\ Immunology and Institute of Laboratory Clinical Diagnostics, School of Medicine, Jiangsu University, Zhenjiang, \\ Jiangsu 212013; ${ }^{3}$ Department of General Surgery, Pucheng Hospital, Weinan, Shaanxi 715500, P.R. China
}

Received September 29, 2019; Accepted July 14, 2020

DOI: $10.3892 / \mathrm{mmr} .2020 .11470$

\begin{abstract}
Non-coding RNAs (ncRNAs) have been previously reported to serve an important role in transcription. In addition, several studies have revealed that long ncRNAs (lncRNAs) have a crucial role in human diseases. However, the association between IncRNAs and inflammation-induced intestinal macrophages in the intestinal mucosal barrier has remained elusive. In the present study, intestinal macrophages from healthy Sprague Dawley rats were divided into two groups: The experimental group, consisting of intestinal macrophages treated with $1 \mathrm{mg} / 1$ lipopolysaccharide (LPS) and the control group, composed of untreated cells. Differentially expressed (DE) lncRNAs and mRNAs between the control and experimental groups were identified using microarray profiling. The levels of DE mRNAs and lncRNAs were measured by reverse transcription-quantitative PCR (RT-qPCR). Furthermore, Gene Ontology (GO) and pathway enrichment analyses of DE mRNAs and lncRNAs were performed. To identify core regulatory factors among DE lncRNAs and mRNAs, a IncRNA-mRNA network was constructed. A total of 357 DE lncRNAs and 542 DE mRNAs between the LPS-treated and untreated groups were identified (fold-change $>1.5 ; \mathrm{P}<0.05$ ). In addition, selected microarray data were confirmed by RT-qPCR. GO analysis of the DE mRNAs indicated that the biological functions of the upregulated mRNAs included inflammatory response, immune response, metabolic process and signal
\end{abstract}

Correspondence to: Professor Shengchun Dang, Department of General Surgery, The Affiliated Hospital, Jiangsu University, 438 Liberation of North Road, Zhenjiang, Jiangsu 212001, P.R. China E-mail: dscgu@163.com

*Contributed equally

Abbreviations: LPS, lipopolysaccharide; lncRNA, long non-coding RNA; IBD, intestinal barrier dysfunction

Key words: long non-coding RNA, mRNA, intestinal macrophages, expression profiles transduction, whereas those of the downregulated mRNAs were metabolic process, cell cycle, apoptosis and inflammatory response. In addition, pathway enrichment analysis of the upregulated mRNAs revealed that the most enriched pathways were the NF- $\mathrm{NB}$ signaling pathway, B-cell receptor signaling pathway and apoptosis, while the downregulated mRNAs were significantly involved in metabolic pathways, the phosphatidylinositol signaling system, cytokine-cytokine receptor interaction and the Toll-like receptor signaling pathway. The 1ncRNA-mRNA co-expression network suggested that lncRNAs NONMMUT024673 and NONMMUT062258 may have an important role in LPS-induced intestinal macrophages. The present study identified the DE profiles between LPS- and non-LPS-treated intestinal macrophages. These DE lncRNAs and mRNAs may be used as potential targets for attenuating excessive inflammatory response in intestinal mucosal barrier dysfunction.

\section{Introduction}

Human intestinal barriers are important in facilitating the absorption of nutrients and the prevention of bacterial invasion (1). However, numerous pathological conditions, including severe acute pancreatitis (2), inflammatory bowel disease (3) and infectious diarrheal syndromes, may lead to intestinal barrier dysfunction (IBD). Furthermore, macrophages have an important role in IBD. One of the major pathophysiological changes in IBD is the overactivation of the inflammatory response, with intestinal macrophages acting as the most important effector cells in this response. Macrophages are the core cells that initiate and regulate the inflammatory reaction, and therefore, their activation and dysfunction are associated with IBD development (4).

Protein-coding genes only represent a small portion of the human genome $(<2 \%)$, while the majority of the genome is transcribed into non-coding RNAs (ncRNAs) (5-7). Long ncRNA (lncRNA) transcripts lack significant open reading frames and are composed of molecules $>200$ nucleotides in length (8). Initially, IncRNAs were considered as transcriptional artifacts; however, they were then revealed to have important regulatory roles that remain to be fully elucidated in detail. With the development of lncRNA microarrays, high-throughput sequencing 
and bioinformatics, an increasing number of lncRNAs have been identified. LncRNAs have attracted considerable attention in the research fields of molecular biology and biomedical science. Functionally, lncRNAs regulate gene expression at the epigenetic, transcriptional and post-transcriptional levels and are involved in several biological processes, including genomic imprinting, chromosome dosage compensation, $\mathrm{X}$ chromosome silencing, chromosome modification, intra-nuclear transport and transcriptional activation $(4,9)$. Recent studies have suggested that lncRNAs participate in various complex human diseases, including aging, hematopoiesis, neurobiology, cancer, muscle biology and immunology (10-16). Several studies have indicated that 1 ncRNA H19 may regulate the intestinal epithelial barrier $(17,18)$, whereas the expression of lncRNA cyclin-dependent kinase inhibitor 2B antisense 1 has been associated with inflammatory bowel disease and intestinal barrier formation (19).

Macrophages have been considered to be important effectors of the inflammatory response, which in turn may be regulated by IncRNAs $(20,21)$. However, the potential association between lncRNAs and macrophages/monocytes in post-IBD has remained to be fully investigated. Therefore, in the present study, an in vitro cell model was established to investigate the expression profiles of lncRNAs and mRNAs in macrophages. The aim of the present study was to determine the expression profiles of lncRNAs and mRNAs in lipopolysaccharide (LPS)-induced rat macrophages and to provide a direction for further studies.

\section{Materials and methods}

Materials. A total of six Sprague-Dawley (SD) rats (female and male, 6-8 weeks, weight, 200-250 g) were provided by the Yangzhou University Experimental Animal Center. RPMI-1640 medium, fetal bovine serum (FBS), penicillin and streptomycin were purchased from Hyclone (Cytiva). Isotonic cell separation solution (Percoll solution) and collagenase IV were obtained from Worthington Biochemical Corp. The primary rabbit polyclonal antibody against CD14 (cat. no. AF2128; Beyotime Institute of Biotechnology) was diluted at 1:300 prior to use, whereas the secondary FITC-conjugated goat anti-rabbit IgG $(\mathrm{H}+\mathrm{L})$ (Beyotime Institute of Biotechnology) was diluted at 1:50.

Furthermore, total RNA was extracted with a TRIzol reagent (Invitrogen; Thermo Fisher Scientific, Inc.) according to the manufacturer's protocol. RNA quantity and quality were measured using the NanoDrop ND-1000 spectrophotometer (PeqLab). RNA integrity was evaluated by agarose gel electrophoresis on the Agilent 2100 Bioanalyzer (Agilent Technologies, Inc.). In the present study, the following materials were also used: Primers/probes; TRIzol reagent (cat. no. 15596-026); diethyl pyrocarbonate-treated $\mathrm{H}_{2} \mathrm{O}$ (cat. no. 750024), SuperScriptIII reverse transcriptase (cat. no. R250-01); SYBR-Green I (cat. no. CS7561); oligo Dt/random primers/specific primers; Platinum Taq DNA polymerase (cat. no. 10966034); $100 \mathrm{mM}$ deoxyribonucleoside triphosphates (cat. no. 18427013; all from Invitrogen; Thermo Fisher Scientific, Inc.); miRNAeasy Mini kit (cat. no. 217184; Qiagen); avantin; and RNase inhibitor (cat. no. E00381; Fermentas; Thermo Fisher Scientific, Inc.).
Isolation of intestinal macrophages. SD rats were housed in individual cages and maintained at $21-23^{\circ} \mathrm{C}$ and $60 \pm 10 \%$ humidity on a $12 \mathrm{~h}$ light/dark schedule. The rats were acclimatized for one week prior to any experimental procedure and had free access to standard rat chow and water. All animal experiments were approved by the Ethics Committee of the Affiliated Hospital of Jiangsu University (Zhenjiang, China) and were performed in compliance with the guidelines specified by the Institutional Ethics Board. All animal experiments were also in compliance with the Animal Research: Reporting of In Vivo Experiments guidelines $(22,23)$. All efforts were made to minimize the number of animals used and their suffering. Rats were anesthetized with an intraperitoneal injection of $40 \mathrm{mg} / \mathrm{kg}$ sodium pentobarbital and were subsequently dissected to remove the whole intestine. Following the isolation of the entire small intestine from a rat by a 5-min experimental procedure, the SD rats were sacrificed by decapitation. The experimental time for isolation of intestinal macrophages from the six rats was 1 week. Each rat was processed individually for sampling. Intestines were opened longitudinally, washed with cold PBS ( $\mathrm{pH}$ 7.4), placed in Hank's balanced salt solution (HBSS) containing $1 \mathrm{~g} / 1$ EDTA and incubated in a $37^{\circ} \mathrm{C}$ water bath with gentle agitation for $60 \mathrm{~min}$. The supernatant was subsequently discarded, collagenase IV (5 g/l) was added and the tissue was incubated for an additional $2 \mathrm{~h}$. The mixture was poured through a 400-mesh screen filter and subsequently, the resulting cells were washed with HBSS, suspended in $500 \mathrm{~g} / \mathrm{l}$ cell separation isotonic solution (Beyotime Institute of Biotechnology) and centrifuged at $733 \times \mathrm{g}$ at $4^{\circ} \mathrm{C}$ for $15 \mathrm{~min}$. The resulting pellet containing the intestinal macrophages was washed three times with HBSS without calcium and magnesium and cells were stained with trypan blue. The viability of macrophages following treatment was $\sim 85 \%$. Intestinal macrophages were cultured in RPMI-1640 medium supplemented with $10 \mathrm{~g} / 1 \mathrm{FBS}, 100 \mu \mathrm{g} / \mathrm{ml}$ penicillin and $100 \mathrm{U} / \mathrm{ml}$ streptomycin. Following dilution to a density of $5 \times 10^{5}$ cells $/ \mathrm{ml}$, $2 \mathrm{ml}$ and $200 \mu \mathrm{l}$ of macrophage suspension were seeded into 6 and 96-well culture plates, respectively. The plates were then incubated at $37^{\circ} \mathrm{C}$ in a humidified incubator with $5 \% \mathrm{CO}_{2}$. Macrophages were divided into two groups, namely the control group (without LPS) and the experimental group (treated with LPS at a concentration of $1 \mathrm{mg} / \mathrm{l}$ ). A total of three experimental and three control groups were used for repeated experiments, so that that one experimental and one control group with three samples each were used. Following the addition of LPS, cells were cultured for $6 \mathrm{~h}$ and total RNA was extracted. The LPS concentration was selected according to a previous study (24).

Identification of intestinal macrophages. In general, intestinal macrophages lack the expression of the innate immune receptor CD14 and do not secrete pro-inflammatory cytokines in response to commensal bacterial infection. In a previous study, a unique macrophage subset in the human intestine was identified. This macrophage subset secreted larger amounts of CD14-associated pro-inflammatory cytokines compared with typical intestinal resident CD14- CD33 ${ }^{+}$macrophages (25).

For the identification of intestinal macrophages, cells were seeded into 24-well plates and allowed to attach. Once optimal cell growth was achieved (the density was moderate, the cells were connected in a spindle shape, the adhesion was good, 
and the confluence had reached $\sim 95 \%$ ), the culture medium was removed, cells were rinsed twice with PBS for $10 \mathrm{~min}$ and fixed with $1 \mathrm{ml}$ of $4^{\circ} \mathrm{C}$ pre-cooled methanol. Subsequently, cells were incubated with CD14 rabbit polyclonal antibody (dilution, 1:300) overnight at $4^{\circ} \mathrm{C}$. Cells were then incubated with FITC-conjugated goat anti-rabbit $\operatorname{IgG}(\mathrm{H}+\mathrm{L}$; dilution, 1:50) at $37^{\circ} \mathrm{C}$ for $1 \mathrm{~h}$, washed three times with PBS and images were captured under a fluorescence microscope. Green fluorescence indicated a positive signal.

Total RNA extraction. Cells were cultured in the presence of LPS $(1 \mathrm{mg} / \mathrm{ml})$ for $6 \mathrm{~h}$ and total RNA was extracted using a TRIzol reagent according to the manufacturer's protocol. RNA quality was determined using a Nanodrop 2000 Spectrometer (Thermo Fisher Scientific,Inc.) and RNA integrity was assessed by visualization of the $28 \mathrm{~S}$ and $18 \mathrm{~S}$ ribosomal (r)RNA bands on an agarose gel. Following removal of rRNA, the purified RNA was amplified and reverse transcribed into fluorescent complementary (c)DNA using a Quick Amp Labeling kit (Agilent Technologies, Inc.) according to the manufacturer's protocol.

Microarray analysis. GeneChip Mouse Transcriptome Array 1.0 (Affymetrix; Thermo Fisher Scientific, Inc.) is considered as the most powerful and flexible tool for measuring a broad range of expression profiles across the whole mouse transcriptome. Therefore, this tool was used in the present study.

Differentially expressed (DE) IncRNAs and mRNAs demonstrating statistical significances were identified by volcano plot filtering and hierarchical clustering. Subsequently, the purified RNA was reverse-transcribed into cDNA according to the requirements set by GMINIX BioTech. The cDNA was then fragmented, labeled with fluorescent dyes and hybridization was performed in a GeneChip Hybridization Oven 645 (Affymetrix; Thermo Fisher Scientific, Inc.). Following chip washing, arrays were scanned with the GeneChip Scanner (Affymetrix; Thermo Fisher Scientific, Inc.) to determine fluorescence intensity. Imaging data were saved and probe summarization was performed using the Expression Console software (v.1.3.1.187; Affymetrix; Thermo Fisher Scientific, Inc.). The significance analysis of the microarray data was used to identify DE lncRNAs and mRNAs. Following significant and false-discovery rate analyses, the significance threshold for the upregulated and downregulated genes was set to a fold change of $>1.5$ and $\mathrm{P} \leq 0.05$. In the present study, variance was relatively low; therefore, cut-off values were set to $\geq 1.5$-fold and $\leq 0.66$-fold change for upregulated and downregulated genes, respectively $(26,27)$. Fold modification provided more significant insight, with 1.5 considered as a good eliminator of background noise. As the fold change level increased to $\geq 2$, the number of genes significantly decreased.

Gene Ontology (GO) analysis. GO analysis (http://www. geneontology.org) was applied to analyze the function of $\mathrm{DE}$ genes (DEGs). GO is the key functional classification tool associated with the National Center for Biotechnology Information. It organizes genes into hierarchical categories to determine the gene regulatory networks in the categories biological process, molecular function and cellular component.
Table I. Primer sequences used to detect the expression of long non-coding RNA and mRNA by quantitative PCR.

\begin{tabular}{ll}
\hline Primer name & \multicolumn{1}{c}{ Sequences $\left(5^{\prime} \rightarrow 3^{\prime}\right)$} \\
\hline NONMMUT047081-F & TCAATTCCAGGACTCTGGATGC \\
NONMMUT047081-R & GGATGGGTCTTCTAATTGCACC \\
NONMMUT024673-F & TGTTGGGATGTCAGCTCTGC \\
NONMMUT024673-R & TGGGCTGTGACGGACTAAAC \\
MUS-TNF-F & ACCGTCAGCCGATTTGCTAT \\
MUS-TNF-R & CTCCAAAGTAGACCTGCCCG \\
MUS-MALT1-F & CATCCCAAGCTTAAAGCGCC \\
MUS-MALT1-R & TTCATAACCGTGCCCTGCAT \\
MUS-TLR4-F & CTGGGGAGGCACATCTTCTG \\
MUS-TLR4-R & TCAGGTCCAAGTTGCCGTTT \\
MUS-IL15-F & GGGCTGTGTCAGTGTAGGTC \\
MUS-IL15-R & GACAGAGTGCTGTTTGCAAGG \\
$\beta$-actin-F & AGCGAGCATCCCCCAAAGTT \\
$\beta$-actin-R & GGGCACGAAGGCTCATCATT
\end{tabular}

F, forward; R, reverse; IL, interleukin; TNF, tumor necrosis factor; TLR, Toll-like receptor; MALT1, MALT1 paracaspase.

Pathway enrichment analysis. Pathway enrichment analysis was used to determine the significant pathways associated with DEGs according to Kyoto Encyclopedia of Genes and Genomes (KEGG) (http://www.genome.jp/kegg/pathway. html), Biocarta (https://cgap.nci.nih.gov/Pathways/BioCarta_ Pathways) and Reactome databases (https://reactome.org/).

lncRNA-mRNA network. A lncRNA-mRNA network was constructed to identify the interactions between genes and lncRNAs. Pearson's correlation coefficient (PCC) was calculated and the R-value was used to compare the PCC of the correlation between lncRNAs and coding genes. Network structure analysis was further performed to identify the core regulatory factors (genes). Finally, a coding-non-coding gene co-expression network was constructed by selecting the significantly co-expressed lncRNAs and mRNAs.

Reverse transcription-quantitative PCR (RT-qPCR) analysis. Total RNA from treated cells was isolated using TRIzol reagent and reverse-transcribed into cDNA with SuperScript III reverse transcriptase (both from Invitrogen; Thermo Fisher Scientific, Inc.). The expression levels of lncRNAs or mRNAs were detected using RT-qPCR. The specific primers are listed in Table I. IncRNA expression levels were normalized to the expression of $18 \mathrm{~S}$ rRNA according to the double-standard curves method (8). The primers for $\beta$-actin amplification are mentioned in Table I. Calculations were performed in Excel 15.0.5249.1001 (Microsoft Corp.) using equations provided by Applied Biosystems (Thermo Fisher Scientific, Inc.). The SYBR-Green (GMINIX BioTech) dye detection method and a CFX96 ${ }^{\mathrm{TM}}$ Real-Time System (Bio-Rad Laboratories, Inc.) were used to perform qPCR analysis. The qPCR conditions were as follows: $95^{\circ} \mathrm{C}$ for $2 \mathrm{~min}$ and 40 cycles of $10 \mathrm{sec}$ at $95^{\circ} \mathrm{C}, 60^{\circ} \mathrm{C}$ for $30 \mathrm{sec}$ and $70^{\circ} \mathrm{C}$ for $45 \mathrm{sec}$. Melting curve 

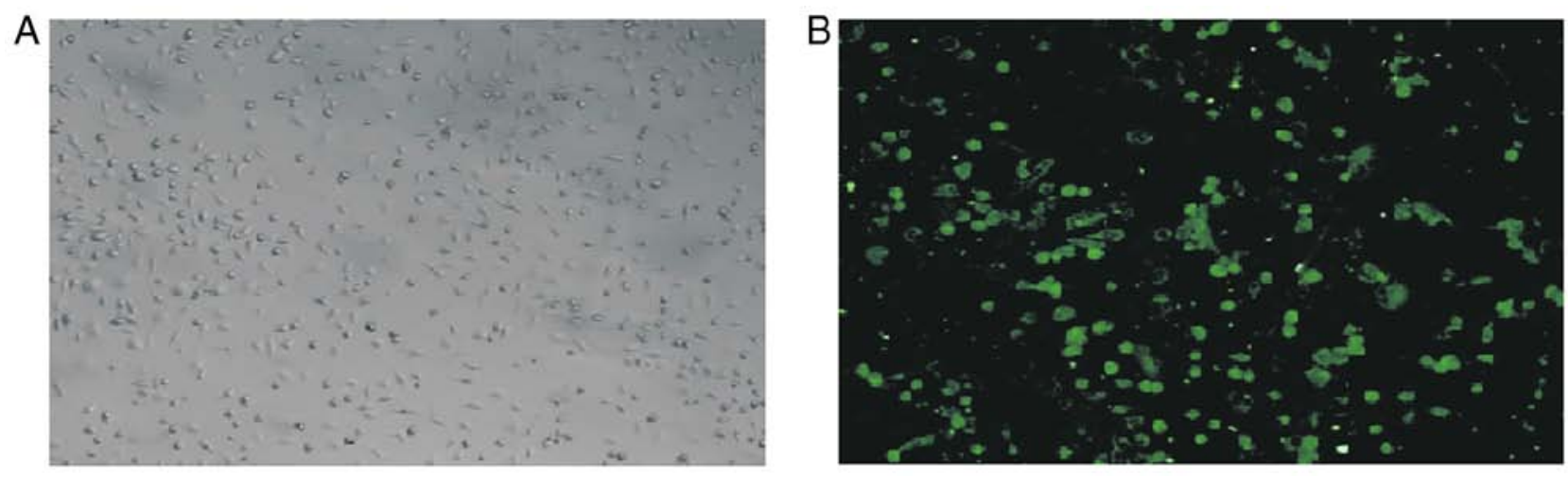

Figure 1. (A) Microscopy image of intestinal macrophages (magnification, x100) cultured for $24 \mathrm{~h}$. (B) Immunofluorescence analysis of CD14-expressing intestinal macrophages (magnification, x200). Positive signals are indicated by green fluorescence.

analysis was performed at $65-95^{\circ} \mathrm{C}$ for $10 \mathrm{sec}$, with a $0.5^{\circ} \mathrm{C}$ increment per read and a hold time of $5 \mathrm{sec}$. Three samples in the experimental group and three samples in the control group were analyzed. All samples were normalized to $\beta$-actin and each experiment was repeated three times.

Statistical analysis. The random variance model (RVM)-modified t-test, combining a two-samples t-test with RVM, was performed to analyze DE IncRNAs and mRNAs using the BRB-arrayTools package (v.4.3.2; Biometrics Research Branch National Cancer Institute). DE lncRNAs were further analyzed by hierarchical clustering using the cluster 3.0 (http://bonsai.hgc.jp/ mdehoon/software/cluster/software. htm) and TreeView tools (http://jtreeview.sourceforge.net/). Furthermore, GO and pathway enrichment analyses were performed and data were analyzed by Fisher's exact test. A paired-samples t-test was used to analyze the RT-qPCR results (GraphPad Prism 5.00; GraphPad Software, Inc.). P $<0.05$ was considered to indicate a statistically significant difference.

\section{Results}

Isolation and identification of intestinal macrophages. Intestinal macrophages were identified by their cellular morphology under a microscope (Fig. 1A). A positive signal for intestinal macrophages was indicated by green fluorescence of FITC-labeled CD14 under a fluorescence microscope (Fig. 1B).

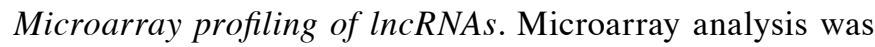
used to examine differences in the expression profiles of IncRNAs and mRNAs between the control macrophage and LPS-treated macrophage groups for the identification of IncRNAs with biological roles during IBD progression. A total of 357 DE lncRNAs were identified with a fold-change of at least 1.5 (Fig. 2). Among the samples from the control group, inter-individual differences were detected by chip testing. Although there were more significant inter-individual differences across samples from the same group, overall, the differences in the control group were more significant than those within the experimental group; therefore, the trend of the overall differences reflected by them was significant. Among these IncRNAs, 245 were upregulated and 112 were downregulated in the LPS-treated macrophage group compared with the control group, with a fold-change threshold of $1.5(\mathrm{P}<0.05$; Tables II and III).

Gene expression profiling by microarray analysis. DE mRNAs were identified following comparison between the LPS-treated and control macrophage groups. A total of 187 upregulated and 355 downregulated mRNAs were obtained with a fold change of $\geq 1.5$ ( $\mathrm{P}<0.05$; Tables IV and V). Hierarchical clustering and volcano plot filtering were utilized to analyze the data (Fig. 2). Thereby, DE mRNAs and IncRNAs were identified between the LPS-treated and control macrophage groups.

GO analysis of DEGs. To determine the roles of DEGs in the categories biological process and molecular function, $\mathrm{GO}$ analysis was performed. A total of $303 \mathrm{GO}$ terms were enriched by the upregulated genes (Fig. 3; P $\leq 0.05$ ), with the most significantly enriched terms being associated with inflammatory responses. Therefore, DE mRNAs were associated with IL-1 $\alpha$, NLR family pyrin domain-containing 3 , lymphocyte antigen 96 and TNF. Furthermore, 366 GO terms were enriched by the downregulated genes, including lipid metabolic processes, inflammatory response, positive regulation of apoptotic processes and immune responses (Fig. 4).

Pathway enrichment analysis of DEGs. Pathway enrichment analysis of DE mRNAs was performed to provide insight into the cellular pathways associated with the selected mRNAs. A total of 61 and 76 pathways were associated with the upregulated and downregulated genes, respectively, the most relevant pathway was 'osteoclast differentiation' (Fig. 5). The results were significant in the LPS-treated macrophage group, the most relevant was 'metabolic pathways' (Fig. 6). These findings suggested that these pathways were associated with the pathogenesis and biochemical characteristics of macrophages involved in IBD.

lncRNA-mRNA network. To identify the IncRNAs and mRNAs that are involved in intestinal macrophage-mediated inflammation, an lncRNA-mRNA interaction network was individually constructed for the LPS-treated and control groups, and their differences were then analyzed. For the coding-non-coding gene co-expression network, five DE lncRNAs and mRNAs 


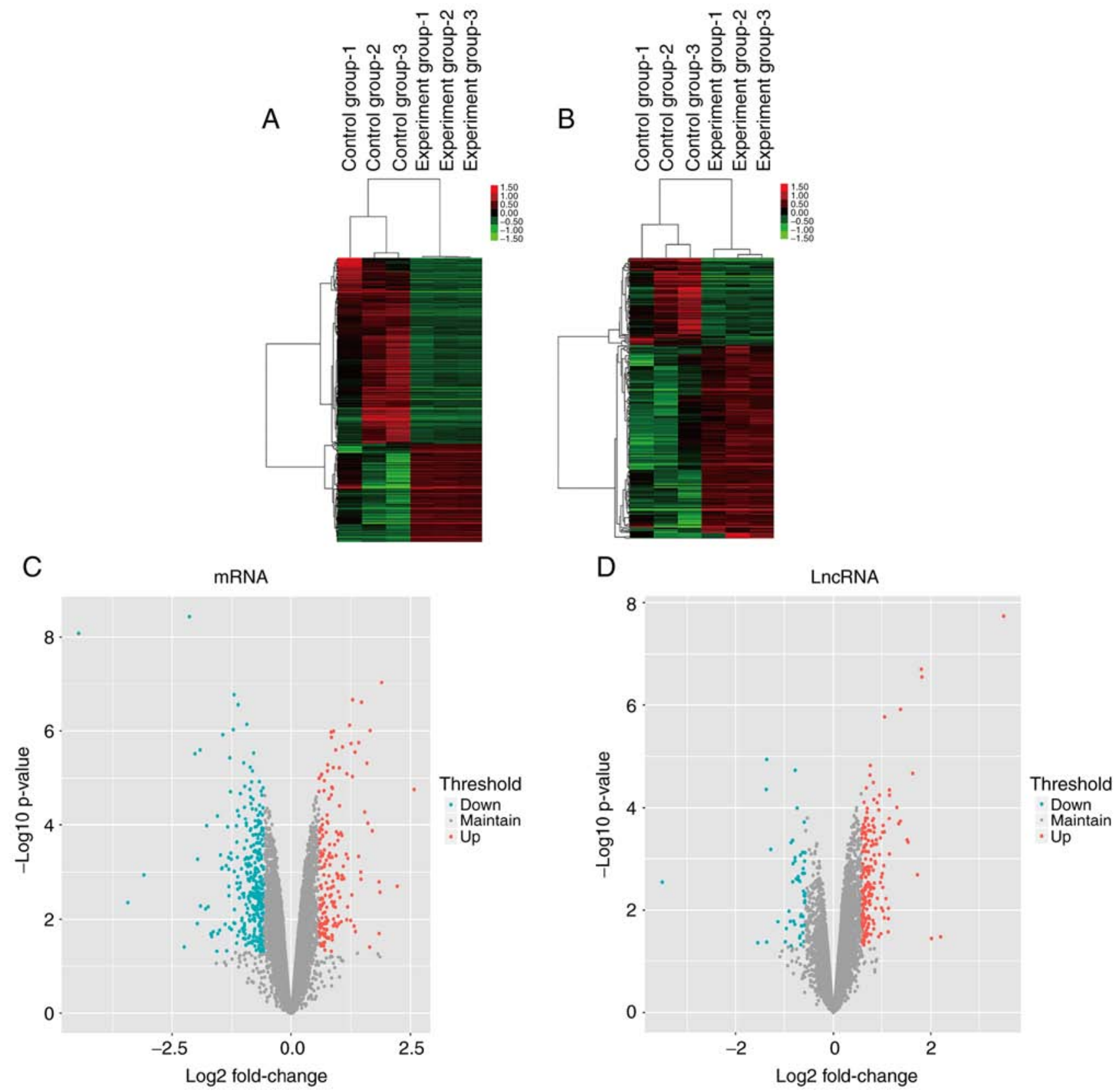

Figure 2. (A) Hierarchical clustering of DE mRNAs. (B) Hierarchical clustering for DE lncRNAs. Red and green indicate high and low relative expression, respectively. (C) Volcano plot of DE mRNAs. (D) Volcano plot of DE lncRNAs. Red and blue indicate high and low relative expression, respectively. DE, differentially expressed; lncRNA, long non-coding RNA; down, downregulated; up, upregulated.

were selected. Furthermore, six lncRNAs and mRNAs were selected by combining the co-expression network results with the microarray data. The results indicated that several lncRNAs, including NONMMUT028346, NONMMUT047081, XR_105830, NONMMUT024673, n-R5s54-F and NONMMUT046238 may serve an important role with the highest degree of interaction in the gene co-expression network (Fig. 7). The aforementioned coding genes were involved in several biological processes, including inflammatory response, apoptosis and cell death.

$R T-q P C R$ verification. To further validate the microarray results, two DE IncRNAs and four DE mRNAs were selected from the lncRNA-mRNA network. Subsequently, RT-qPCR analysis was performed on two additional independent intestinal macrophage groups. The microarray results for the lncRNA and mRNA transcripts were consistent with those obtained using RT-qPCR analysis (Figs. 8 and 9).

\section{Discussion}

Emerging evidence has revealed that thousands of lncRNAs are encoded by the human genome. IncRNAs function as transcriptional and post-transcriptional regulators, may directly act on chromatin-modifying complexes and affect several cellular and developmental pathways (28). The intestinal mucosal barrier is involved in the protection of the human body; however, several pathogenic factors may contribute to IBD (29-32). Among the numerous types of inflammatory cells, macrophages may exert pro-inflammatory and 
Table II. Top 20 upregulated lncRNAs in the macrophage + lipopolysaccharide group compared with the macrophage group. Results were filtered using fold-change $>1.5$ as the threshold.

\begin{tabular}{|c|c|c|c|c|}
\hline Accession no. & P-value & FDR & Fold-change & Chromosome \\
\hline NONMMUT067603 & $1.43 \times 10^{-2}$ & 0.001 & 11.08 & 8 \\
\hline NONMMUT016061 & $2.26 \times 10^{-2}$ & 0.269 & 4.52 & 12 \\
\hline NONMMUT016098 & $2.49 \times 10^{-2}$ & 0.282 & 3.97 & 12 \\
\hline NONMMUT059063 & $1.00 \times 10^{-7}$ & 0.001 & 3.50 & 6 \\
\hline NONMMUT065205 & $1.00 \times 10^{-7}$ & 0.001 & 3.42 & 8 \\
\hline NONMMUT057311 & $9.90 \times 10^{-4}$ & 0.060 & 3.24 & 6 \\
\hline NONMMUT028346 & $9.10 \times 10^{-6}$ & 0.009 & 3.04 & 17 \\
\hline NONMMUT054995 & $1.95 \times 10^{-4}$ & 0.032 & 2.87 & 5 \\
\hline NONMMUT028345 & $9.73 \times 10^{-5}$ & 0.034 & 2.81 & 17 \\
\hline NONMMUT023770 & $2.26 \times 10^{-4}$ & 0.025 & 2.58 & 15 \\
\hline NONMMUT053098 & $9.28 \times 10^{-4}$ & 0.001 & 2.56 & 5 \\
\hline NONMMUT003807 & $2.00 \times 10^{-7}$ & 0.025 & 2.50 & 1 \\
\hline NONMMUT047081 & $1.01 \times 10^{-4}$ & 0.020 & 2.43 & 4 \\
\hline NONMMUT031303 & $4.89 \times 10^{-5}$ & 0.014 & 2.37 & 18 \\
\hline NONMMUT022922 & $2.22 \times 10^{-5}$ & 0.010 & 2.21 & 15 \\
\hline NONMMUT044804 & $2.72 \times 10^{-4}$ & 0.016 & 2.21 & 3 \\
\hline NONMMUT017063 & $1.07 \times 10^{-5}$ & 0.044 & 2.21 & 13 \\
\hline NONMMUT009063 & $2.96 \times 10^{-5}$ & 0.024 & 2.18 & 11 \\
\hline NONMMUT028242 & $6.49 \times 10^{-3}$ & 0.148 & 2.17 & 17 \\
\hline NONMMUT068342 & $7.31 \times 10^{-5}$ & 0.246 & 2.16 & 9 \\
\hline
\end{tabular}

All of these lncRNAs are currently unnamed. lncRNA, long non-coding RNA; FDR, false discovery rate; up, upregulation.

Table III. Downregulated lncRNAs in the macrophage + lipopolysaccharide group compared with the macrophage group. Results were filtered using fold-change $>2$ as the threshold.

\begin{tabular}{lccccc}
\hline Accession no. & P-value & FDR & Fold-change & LncRNA name & Chromosome \\
\hline NR_028499 & $1.52 \times 10^{-3}$ & 0.074 & 11.49 & Fxyd2 & 9 \\
KnowTID_00004330 & $1.00 \times 10^{-7}$ & 0.002 & 5.07 & - & 2 \\
ENSMUST00000117094 & $2.96 \times 10^{-3}$ & 0.102 & 4.55 & Gm11914 & 4 \\
NONMMUT041835 & $1.35 \times 10^{-3}$ & 0.288 & 2.94 & - & 2 \\
NONMMUT046238 & $3.15 \times 10^{-2}$ & 0.314 & 2.94 & - & 4 \\
ENSMUST00000181818 & $2.82 \times 10^{-4}$ & 0.035 & 2.78 & Kans12-ps & 7 \\
NONMMUT065008 & $1.80 \times 10^{-5}$ & 0.013 & 2.63 & - & 8 \\
NONMMUT055602 & $3.90 \times 10^{-6}$ & 0.006 & 2.56 & - & 6 \\
uc029szy.1 & $3.44 \times 10^{-3}$ & 0.110 & 2.56 & - & 17 \\
NONMMUT022580 & $3.08 \times 10^{-2}$ & 0.311 & 2.56 & - & 15 \\
NONMMUT005859 & $3.28 \times 10^{-4}$ & 0.038 & 2.50 & - & 1 \\
NONMMUT000091 & $1.10 \times 10^{-2}$ & 0.193 & 2.22 & - & 11 \\
ENSMUST00000122180 & $4.79 \times 10^{-2}$ & 0.381 & 2.22 & Gm12138 & X \\
NONMMUT052111 & $4.71 \times 10^{-2}$ & 0.378 & 2.08 & - & 15 \\
ENSMUST00000172263 & $8.96 \times 10^{-5}$ & 0.025 & 2.01 & Gm1848 & - \\
NONMMUT024673 & $2.89 \times 10^{-2}$ & 0.302 & 2.06 & & \\
\hline
\end{tabular}

FDR, false discovery rate; lncRNA, long non-coding RNA.

anti-inflammatory properties. Intestinal macrophages in the lamina propria mucosa represent the largest pool of tissue macrophages in the human body. These macrophages have an important role in the regulation of the intestinal barrier 
Table IV. Top 20 upregulated mRNAs in the macrophage + lipopolysaccharide group compared with the macrophage group. The results were filtered using a fold-change of $>1.5$ as the threshold.

\begin{tabular}{|c|c|c|c|c|c|}
\hline Accession no. & $\mathrm{P}$-value & FDR & Fold-change & Gene name & Chromosome \\
\hline NM_022415 & $1.67 \times 10^{-4}$ & 0.170 & 2.47 & Ptges & 2 \\
\hline NM_019472 & $7.00 \times 10^{-7}$ & 0.030 & 2.63 & Myo10 & 15 \\
\hline NM_001145799 & $4.26 \times 10^{-4}$ & 0.002 & 2.64 & Ctla2a & 13 \\
\hline NM_001276764 & $6.24 \times 10^{-4}$ & 0.040 & 2.72 & Dst & 1 \\
\hline NM_008311 & $1.00 \times 10^{-7}$ & 0.049 & 2.72 & Htr $2 b$ & 1 \\
\hline NM_011158 & $1.40 \times 10^{-5}$ & 0.001 & 2.73 & Prkar2b & 12 \\
\hline NM_009690 & $9.00 \times 10^{-7}$ & 0.012 & 2.87 & Cd51 & 3 \\
\hline NM_008491 & $4.34 \times 10^{-5}$ & 0.002 & 2.94 & $\operatorname{Lcn} 2$ & 2 \\
\hline NM_010554 & $2.75 \times 10^{-2}$ & 0.019 & 3.02 & Illa & 2 \\
\hline NM_010329 & $2.00 \times 10^{-7}$ & 0.295 & 3.09 & Pdpn & 4 \\
\hline NM_008768 & $5.71 \times 10^{-5}$ & 0.001 & 3.10 & Orm1 & 4 \\
\hline NM_010766 & $4.04 \times 10^{-2}$ & 0.021 & 3.19 & Marco & 1 \\
\hline ВС019760 & $7.54 \times 10^{-4}$ & 0.352 & 3.49 & Igk & 6 \\
\hline NM_018866 & $1.31 \times 10^{-2}$ & 0.053 & 3.52 & Cxcl13 & 5 \\
\hline NM_010016 & $1.66 \times 10^{-3}$ & 0.208 & 3.55 & $\mathrm{Cd} 55$ & 1 \\
\hline ENSMUST00000109711 & $4.81 \times 10^{-2}$ & 0.076 & 3.57 & Ighv1-2 & 12 \\
\hline NM_008903 & $9.65 \times 10^{-4}$ & 0.382 & 3.57 & Ppap2a & 13 \\
\hline ENSMUST00000103461 & $9.57 \times 10^{-4}$ & 0.058 & 4.59 & Ighv7-3 & 12 \\
\hline NM_138648 & $4.30 \times 10^{-6}$ & 0.063 & 5.86 & Olr1 & 6 \\
\hline ВC055906 & $2.80 \times 10^{-2}$ & 0.298 & 7.55 & Igk & 6 \\
\hline
\end{tabular}

FDR, false discovery rate.

Table V. Top 20 downregulated mRNAs in the macrophage + lipopolysaccharide group compared with the macrophage group. The results were filtered using a fold-change $>1.5$.

\begin{tabular}{|c|c|c|c|c|c|}
\hline Accession no. & P-value & FDR & Fold-change & Gene name & Chromosome \\
\hline ENSMUST00000081924 & $1.71 \times 10^{-5}$ & 0.007 & 22.22 & Ifitm6 & 7 \\
\hline NM_133661 & $2.48 \times 10^{-3}$ & 0.093 & 10.87 & Slc6a12 & 6 \\
\hline NM_009801 & $5.58 \times 10^{-4}$ & 0.046 & 8.33 & Car2 & 3 \\
\hline NM_001145959 & $2.74 \times 10^{-2}$ & 0.294 & 4.76 & Ndrg2 & 14 \\
\hline NM_008969 & $2.20 \times 10^{-6}$ & 0.003 & 4.35 & Ptgs1 & 2 \\
\hline NM_011198 & $1.00 \times 10^{-6}$ & 0.002 & 4.21 & Ptgs2 & 1 \\
\hline NM_001204203 & $2.57 \times 10^{-4}$ & 0.035 & 4.61 & Spp1 & 5 \\
\hline NM_008361 & $6.87 \times 10^{-3}$ & 0.153 & 4.93 & $\mathrm{Illb}$ & 2 \\
\hline NM_001038614 & $8.00 \times 10^{-7}$ & 0.002 & 3.85 & Olfm1 & 2 \\
\hline NM_001134475 & $2.59 \times 10^{-3}$ & 0.096 & 3.75 & Vcan & 13 \\
\hline NM_008035 & $4.42 \times 10^{-5}$ & 0.020 & 3.45 & Folr2 & 7 \\
\hline NM_016710 & $3.50 \times 10^{-3}$ & 0.111 & 3.45 & Hmgn5 & $X$ \\
\hline NM_008278 & $3.24 \times 10^{-3}$ & 0.107 & 3.33 & Hpgd & 8 \\
\hline NM_011311 & $1.33 \times 10^{-2}$ & 0.209 & 3.23 & S100a4 & 3 \\
\hline NM_017391 & $1.35 \times 10^{-2}$ & 0.210 & 3.23 & Slc5a3 & 16 \\
\hline NM_009250 & $1.56 \times 10^{-2}$ & 0.225 & 3.23 & Serpini1 & 3 \\
\hline NM_148933 & $1.23 \times 10^{-2}$ & 0.202 & 3.13 & Slco4a1 & 2 \\
\hline NM_009644 & $1.57 \times 10^{-5}$ & 0.013 & 2.94 & Ahrr & 13 \\
\hline NM_010330 & $1.20 \times 10^{-2}$ & 0.013 & 2.94 & $\mathrm{Emb}$ & 13 \\
\hline NM_178767 & $3.48 \times 10^{-2}$ & 0.330 & 2.94 & Agmo & 12 \\
\hline
\end{tabular}

FDR, false discovery rate. 


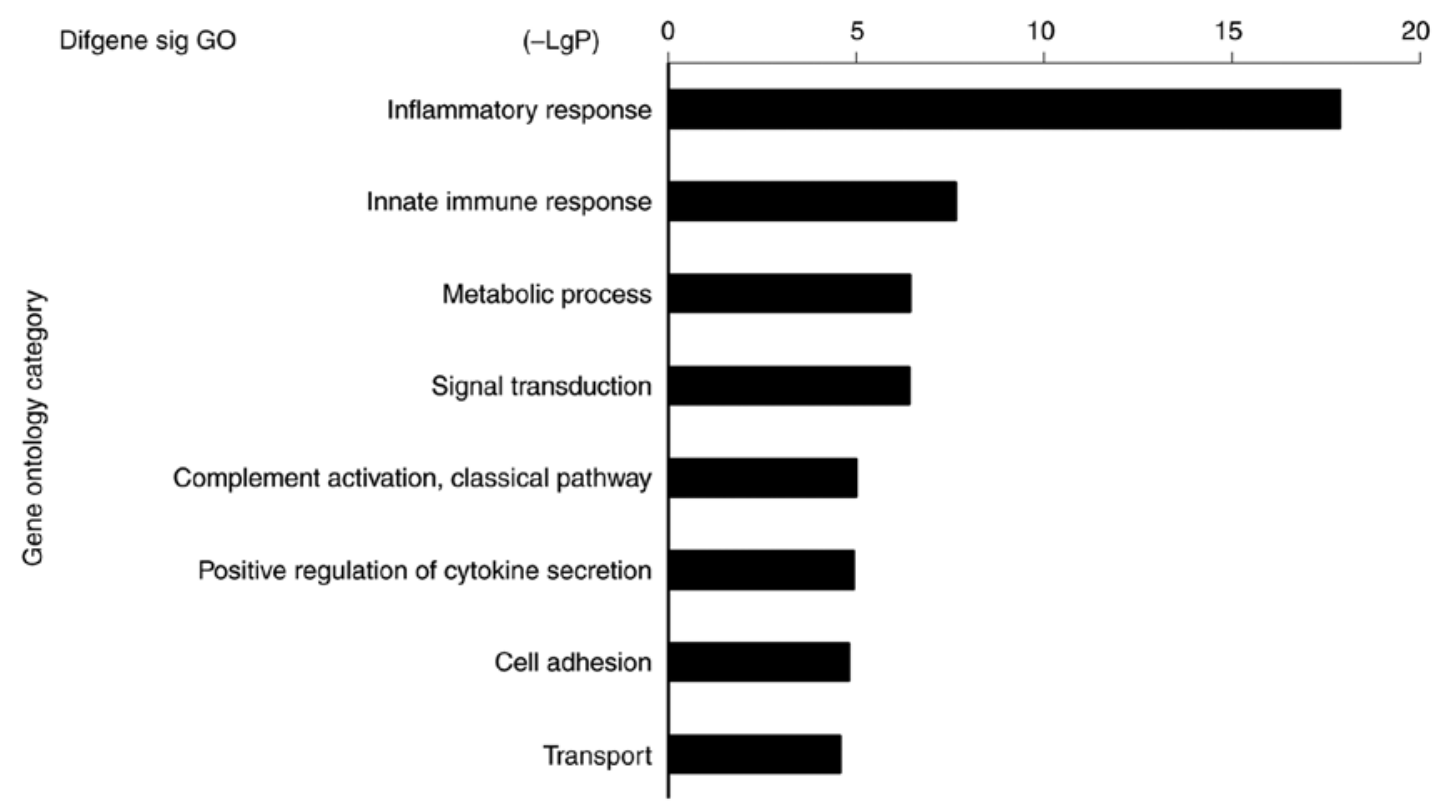

Figure 3. GO terms enriched by upregulated genes are presented $(-\operatorname{LgP}>4.5)$. A larger- $\mathrm{LgP}$ number indicates a smaller P-value for the difference and therefore a more significant association of the particular GO term with the group of genes. The bar graphs represent the enrichment of these genes. GO, Gene Ontology; -LgP, negative natural logarithm of P-value; Sig, significant.

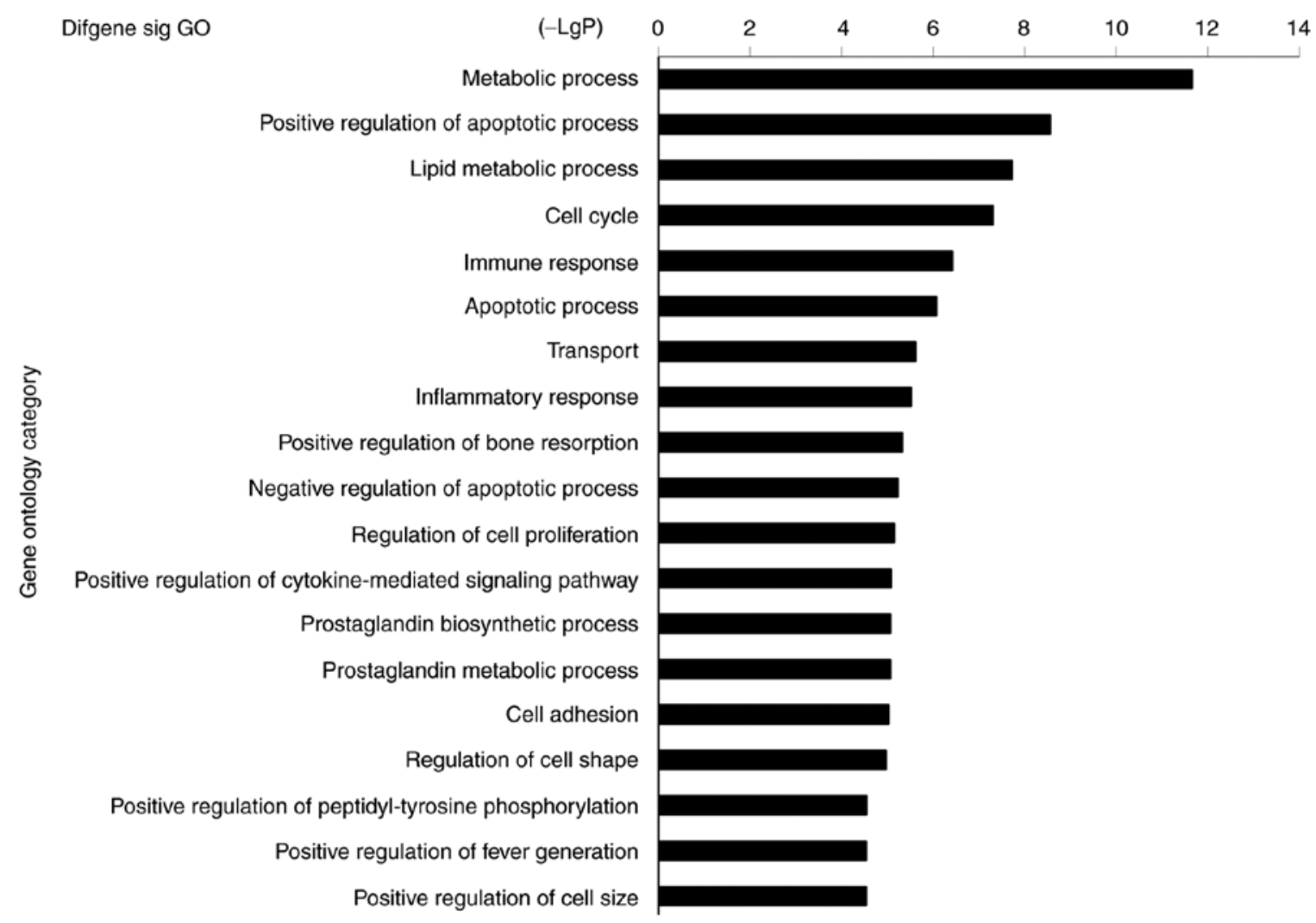

Figure 4. GO terms enriched among downregulated genes are presented (-LgP >4.5). GO, Gene Ontology; -LgP, negative natural logarithm of P-value; Sig, significant.

function. Studies have indicated that intestinal macrophages inhibit overactivation of the inflammatory response to protect the intestinal mucosal barrier $(33,34)$. Several immunological and non-immunological components contribute to intestinal barrier function. The epithelial barrier is one of the most important non-immunological components. A previous study by our group demonstrated that the expression of triggering receptor expressed on myeloid cells-1 (TREM-1) was downregulated by its selective inhibitor LP17 in intestinal macrophages, whereas TREM-1 expression inhibited the 


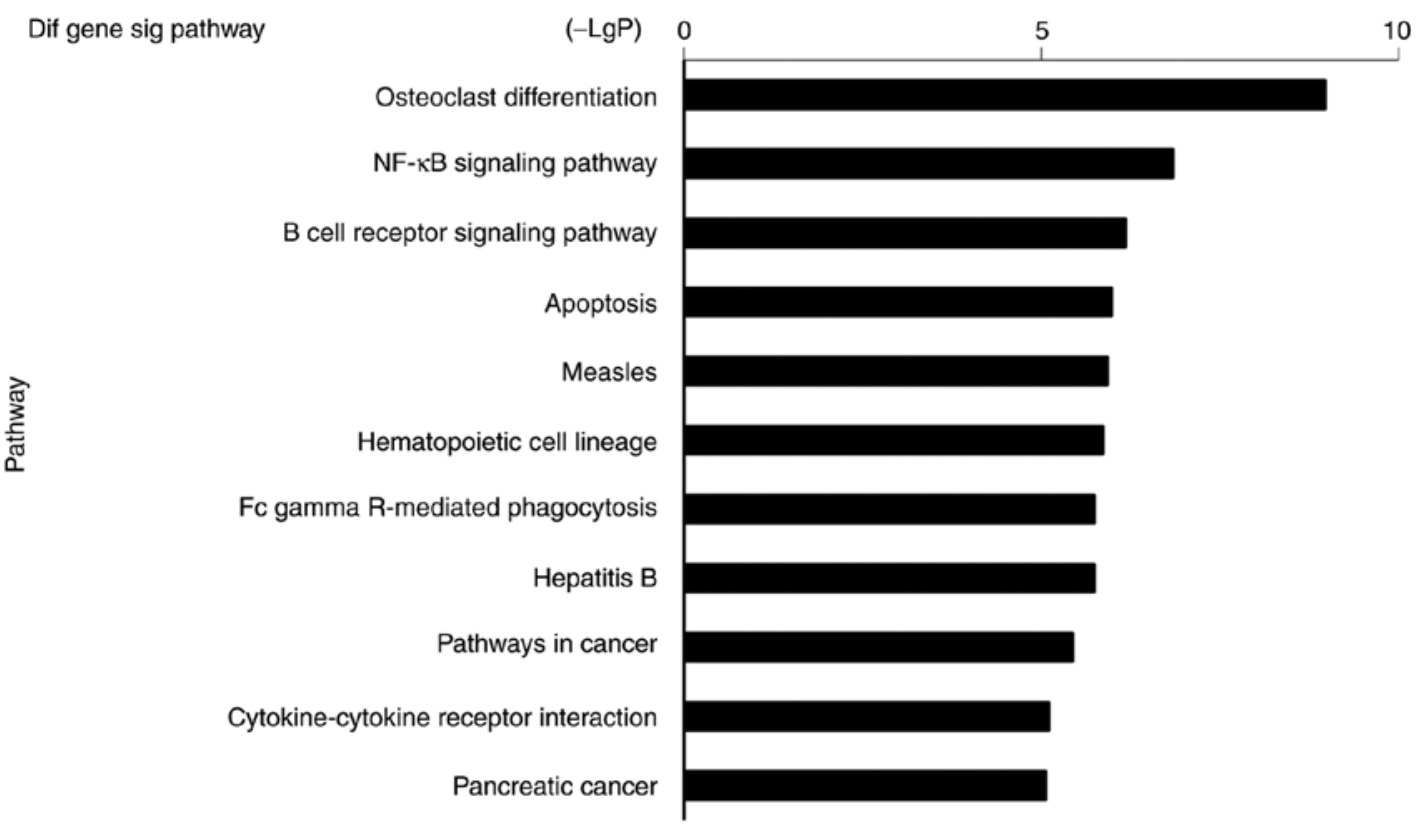

Figure 5. Upregulated pathways are represented (-LgP >5.0). -LgP, negative natural logarithm of P-value; Sig, significant.

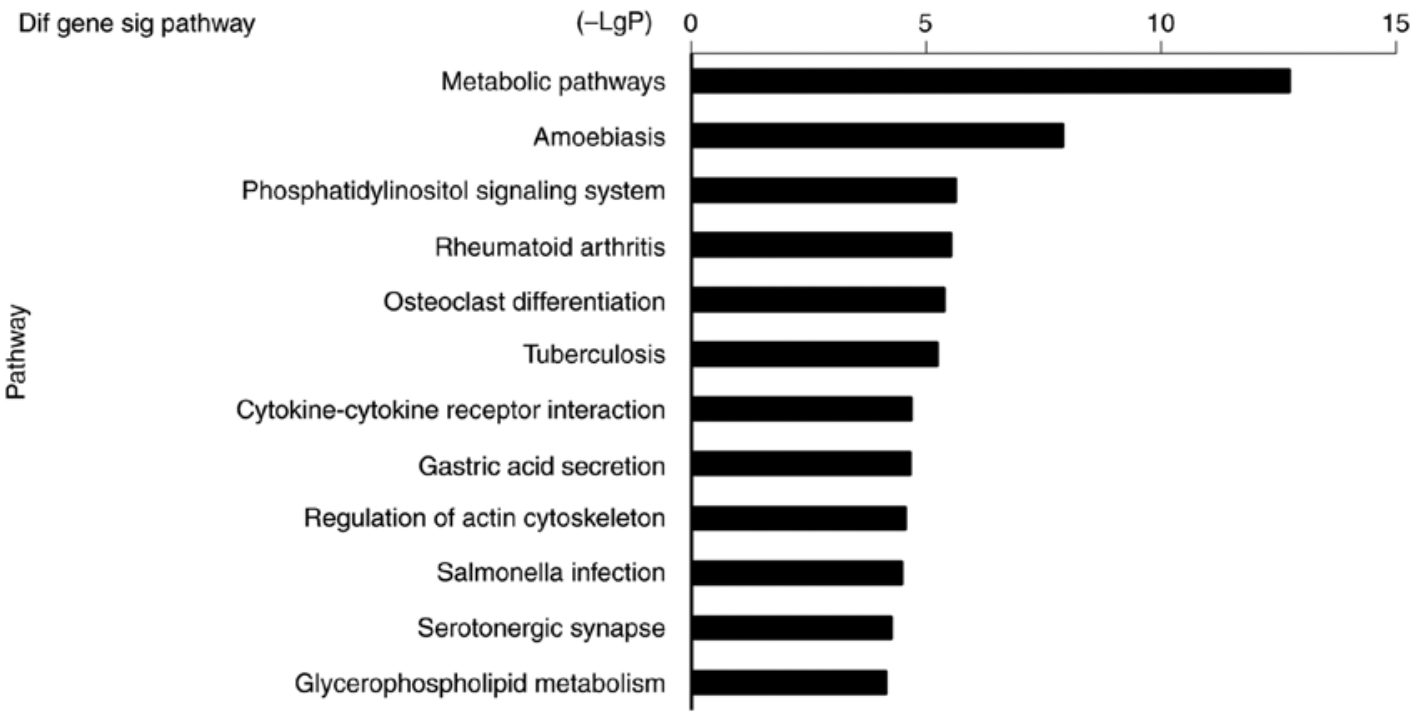

Figure 6. Downregulated pathways are represented (-Lgp >4.0). -LgP, negative natural logarithm of P-value; Sig, significant.

invasive ability of intestinal macrophages into the intestinal epithelium. Therefore, TREM-1 may be considered as a novel target for the treatment of IBD (28). In the present study, cells were analyzed from SD rats, but the microarray kit and the PCR primers used were specifically for mice. We have optimized our PCR primer with the aforementioned sequence, and it worked and amplified the target sequence efficiently, so therefore in our opinion, this primer sequence can be used for the amplification of genes from SD rat cells.

LncRNAs serve as transcriptional and post-transcriptional regulators and may bind to chromatin-modifying complexes to guide them to specific locations. In addition, lncRNAs have been reported to affect several cellular and developmental pathways. Therefore, it is not surprising that the dysregulation of IncRNAs appears to be a significant feature of several complex human diseases. Although rapid progress has been made in associated research fields, the role of IncRNAs in the regulation of inflammatory responses is only now becoming apparent. Carpenter et al (35) confirmed that murine lncRNA cytochrome $\mathrm{C}$ oxygenase II interacted with heterogeneous nuclear ribonucleoproteins to facilitate the activation and inhibition of immune response-associated genes. Cui et al (36) also demonstrated that lncRNA IL-7 receptor (IL7R), overlapping with the 3'-untranslated region of the human IL7R $\alpha$-subunit gene, contributed to the regulation of inflammatory responses. Furthermore, downregulation of lncRNA nuclear paraspecle assembly transcript 1 attenuated the inflammatory response in IBD via modulating the intestinal epithelial barrier and exosome-mediated polarization of macrophages (37). 


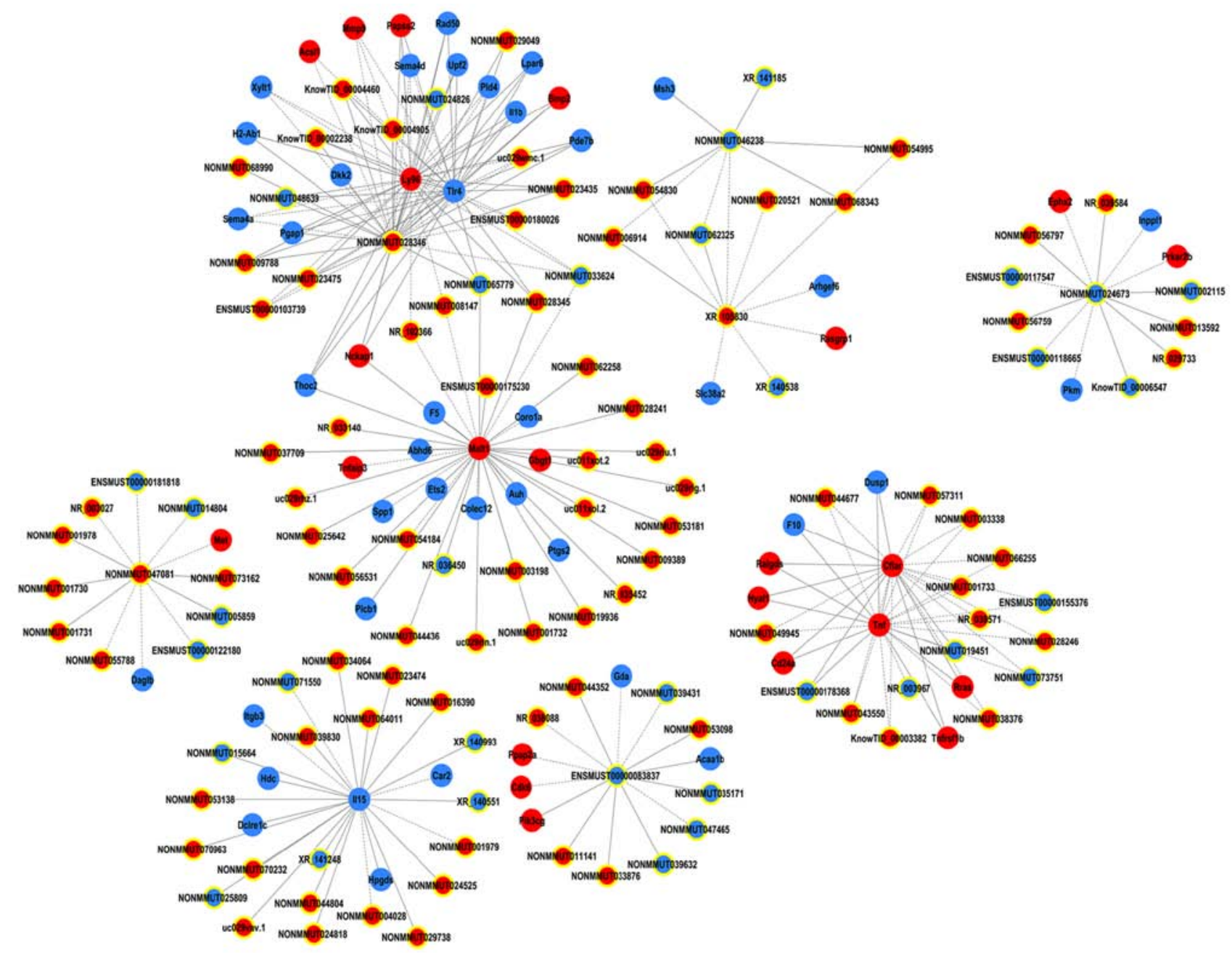

Figure 7. IncRNA-mRNA network. IncRNAs are represented by green circles and all other circles represent mRNAs. Red denotes upregulation and blue downregulation. The solid lines represent positive correlations and the dashed lines represent negative correlations. A total of six significantly DE coding genes and six significantly DE lncRNAs were selected to construct the lncRNA-mRNA network. IncRNA, long non-coding RNA; DE, differentially expressed.

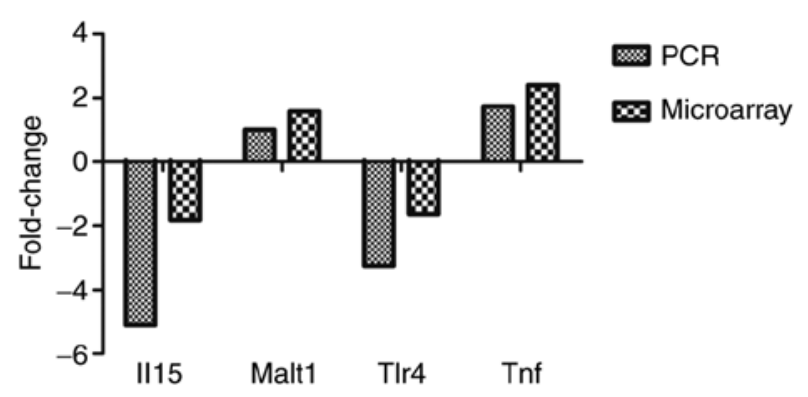

Figure 8. Comparison between microarray and RT-qPCR results. DE mRNAs $(\mathrm{n}=4)$ were validated using RT-qPCR. The columns represent the absolute fold change between the LPS- and non-LPS-treated groups as detected by microarray and RT-qPCR analysis. The RT-qPCR results were closely associated with the microarray data. DE, differentially expressed; LPS, lipopolysaccharide; RT-qPCR, reverse transcription-quantitative PCR; IL, interleukin; TNF, tumor necrosis factor; TLR, Toll-like receptor; MALT1, MALT1 paracaspase.

The present study aimed to investigate the differential expression profiles of IncRNAs between non-LPS and LPS-treated intestinal macrophages. Several lncRNAs were identified in intestinal macrophages, whose expression was

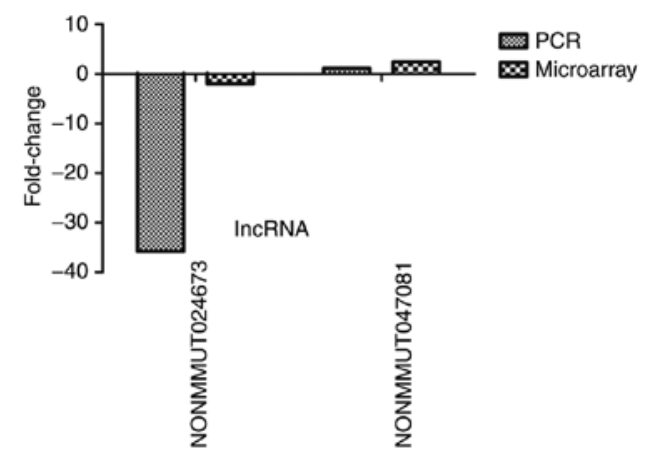

Figure 9. Comparison between microarray and RT-qPCR results. DE lncRNAs $(\mathrm{n}=2)$ were validated using RT-qPCR. The columns represent the absolute fold-change between the LPS- and non-LPS-treated groups as detected by microarray and RT-qPCR analysis. The RT-qPCR results were closely associated with the microarray data. DE, differentially expressed; lncRNA, long non-coding RNAs; LPS, lipopolysaccharide; RT-qPCR, reverse transcription-quantitative PCR.

altered in response to LPS treatment. The RT-qPCR and microarray analyses were used to validate the expression profiles of lncRNAs. The results indicated that lncRNAs in intestinal macrophages were able to regulate the inflammatory 
response to LPS. Furthermore, GO analysis revealed that the upregulated coding genes were predominantly involved in inflammatory and innate immune responses, while the downregulated ones were involved in the positive regulation of the apoptotic process, and immune and inflammatory responses. In addition, pathway enrichment analysis suggested that inflammation-associated pathways, including the NF- $\kappa \mathrm{B}$ and Toll-like receptor (TLR) signaling pathways, were enriched in the upregulated transcripts associated with rheumatoid arthritis. Ma et al (38) indicated that lncRNA $\mathrm{TNF}-\alpha$-induced protein 3 , as a regulator of $\mathrm{NF}-\kappa \mathrm{B}$, modulates inflammation-associated gene transcription in mouse macrophages.

Studies have also demonstrated that lncRNAs may serve a vital role in regulating the transcription of neighboring coding genes (39). The present study predicted that lncRNAs NONMMUT024673 and NONMMUT047081 are able to regulate the inflammatory response via modulating the expression of coding genes. Therefore, bioinformatics analysis may be useful in detecting target genes. Furthermore, the lncRNA-mRNA network revealed that IncRNA NONMMUT028346 interacted with the TLR4 gene. TLR4 is involved in several inflammatory pathways. Based on the co-expression network and microarray data, lncRNAs NONMMUT047081 and NONMMUT024673 were selected to further evaluate their predicted value in LPS-induced intestinal macrophages-mediated inflammatory responses. Therefore, RT-qPCR analysis was performed. The relative expression levels of IncRNAs NONMMUT024673 and NONMMUT047081 were significantly downregulated and upregulated, respectively, in the LPS-treated intestinal macrophage group compared with the control group.

In conclusion, in the present study, DE lncRNAs between LPS-treated and untreated rat intestinal macrophages were identified. The genomic data obtained in the present study may improve the understanding of the role of lncRNAs in the intestinal macrophage-mediated induction of inflammatory responses. However, further studies are required to elucidate the exact regulatory mechanisms of these DE lncRNAs. In addition, the microarray results provided a novel mechanism of macrophage-mediated regulation of IBD.

\section{Acknowledgements}

Not applicable.

\section{Funding}

This study was supported by a grant from the Six Talent Peaks Project in Jiangsu Province (grant no. 2016-WSN-007), the Jiangsu 333 Project Foundation (grant no. BRA2017560) and the Zhenjiang Science and Technology Committee (grant no. SH 2019061). The funding bodies had no role in the design of the study, the collection, analysis and interpretation of data or in writing the manuscript.

\section{Availability of data and materials}

All data generated or analyzed during this study are included in this published article.

\section{Authors' contributions}

SD and LL conceived and supervised the study; LL, SC, QN, $\mathrm{XX}, \mathrm{XW}$ and SD designed the experiments; QN, JC, JQ and LC performed the experiments and had a major role in the acquisition of data; LC and SD analyzed the data; SD, QN, LL and SC, QN, XX, XW revised the manuscript for intellectual content, designed the figures, contributed to interpreting the data and wrote the manuscript with input from all authors. All authors read and approved the final manuscript.

\section{Ethics approval and consent to participate}

All protocols for animal experimentation were approved by the Institutional Animal Care and Use Committees of Jiangsu University (Zhenjiang, China) and experiments were performed according to the Regulation on Animal Experimentation at Jiangsu University (Zhenjiang, China).

\section{Patient consent for publication}

Not applicable.

\section{Competing interests}

The authors declare that they have no competing interests.

\section{References}

1. Shen L, Su L and Turner JR: Mechanisms and functional implications of intestinal barrier defects. Dig Dis 27: 443-449, 2009.

2. Xu GF, Guo M, Tian ZQ, Wu GZ, Zou XP and Zhang WJ: Increased of serum high-mobility group box chromosomal protein 1 correlated with intestinal mucosal barrier injury in patients with severe acute pancreatitis. World J Emerg Surg 9: 61, 2014.

3. Lee SH: Intestinal permeability regulation by tight junction: Implication on inflammatory bowel diseases. Intest Res 13: $11-18,2015$

4. Zhang JX, Wang K, Mao ZF, Fan X, Jiang DL, Chen M, Cui L, Sun K and Dang SC: Application of liposomes in drug development-focus on gastroenterological targets. Int J Nanomedicine 8: 1325-1334, 2013

5. ENCODE Project Consortium: An integrated encyclopedia of DNA elements in the human genome. Nature 489: 57-74, 2012.

6. Dunne MC and Barnes DA: Schematic modelling of peripheral astigmatism in real eyes. Ophthalmic Physiol Opt 7: 235-239, 1987.

7. Derrien T, Johnson R, Bussotti G, Tanzer A, Djebali S, Tilgner H, Guernec G, Martin D, Merkel A, Knowles DG, et al: The GENCODE v7 catalog of human long noncoding RNAs: Analysis of their gene structure, evolution, and expression. Genome Res 22: 1775-1789, 2012.

8. Song X, Cao G, Jing L, Lin S, Wang X, Zhang J, Wang M, Liu $\mathrm{W}$ and Lv C: Analysing the relationship between lncRNA and protein-coding gene and the role of IncRNA as ceRNA in pulmonary fibrosis. J Cell Mol Med 18: 991-1003, 2014.

9. Wilusz JE, Sunwoo H and Spector DL: Long noncoding RNAs: Functional surprises from the RNA world. Genes Dev 23: 1494-1504, 2009.

10. Nakagawa T, Endo H, Yokoyama M, Abe J, Tamai K, Tanaka N, Sato I, Takahashi S, Kondo T and Satoh K: Large noncoding RNA HOTAIR enhances aggressive biological behavior and is associated with short disease-free survival in human non-small cell lung cancer. Biochem Biophys Res Commun 436: 319-324, 2013.

11. Ponting CP, Oliver PL and Reik W: Evolution and functions of long noncoding RNAs. Cell 136: 629-641, 2009.

12. Moran VA, Perera RJ and Khalil AM: Emerging functional and mechanistic paradigms of mammalian long non-coding RNAs. Nucleic Acids Res 40: 6391-6400, 2012. 
13. Sun M and Kraus WL: From discovery to function: The expanding roles of long non-coding RNAs in physiology and disease. Endocr Rev 36: 25-64, 2015.

14. Satpathy AT and Chang HY: Long noncoding RNA in hematopoiesis and immunity. Immunity 42: 792-804, 2015.

15. Kim J, Kim KM, Noh JH, Yoon JH, Abdelmohsen K and Gorospe M: Long noncoding RNAs in diseases of aging. Biochim Biophys Acta 1859: 209-221, 2016.

16. Kung JT, Colognori D and Lee JT: Long noncoding RNAs: Past, present, and future. Genetics 193: 651-669, 2013

17. Chen SW, Wang PY, Liu YC, Sun L, Zhu J, Zuo S, Ma J, Li TY, Zhang JL, Chen GW, et al: Effect of long noncoding RNA H19 overexpression on intestinal barrier function and its potential role in the pathogenesis of ulcerative colitis. Inflamm Bowel Dis 22: 2582-2592, 2016.

18. Zou T, Jaladanki SK, Liu L, Xiao L, Chung HK, Wang JY, Xu Y, Gorospe M and Wang JY: H19 long noncoding RNA regulates intestinal epithelial barrier function via MicroRNA 675 by interacting with RNA-binding protein HuR. Mol Cell Biol 36 : 1332-1341, 2016.

19. Rankin CR, Lokhandwala ZA, Huang R, Pekow J, Pothoulakis C and Padua D: Linear and circular CDKN2B-AS1 expression is associated with inflammatory bowel disease and participates in intestinal barrier formation. Life Sci 231: 116571, 2019.

20. Carpenter $S$ and Fitzgerald KA: Transcription of inflammatory genes: Long noncoding RNA and beyond. J Interferon Cytokine Res 35: 79-88, 2015.

21. Marques-Rocha JL, Samblas M, Milagro FI, Bressan J, Martinez JA and Marti A: Noncoding RNAs, cytokines, and inflammation-related diseases. FASEB J 29: 3595-3611, 2015

22. Kilkenny C, Browne WJ, Cuthi I, Emerson M and Altman DG: Improving bioscience research reporting: The ARRIVE guidelines for reporting animal research. Vet Clin Pathol 41: 27-31, 2012.

23. McGrath JC, Drummond GB, McLachlan EM, Kilkenny C and Wainwright CL: Guidelines for reporting experiments involving animals: The ARRIVE guidelines. Br J Pharmacol 160: 1573-1576, 2010.

24. Zhang JX, Wang K, Zhu WR, Shen Y, Wang PJ and Dang SC: Effect of TREM-1 expression in intestinal macrophages on their invasion and proliferation of intestinal epithelial cells. Shijie Huaren Xiaohua Zazhi 21: 471-477, 2013 (In Chinese).

25. Kamada N, Hisamatsu T, Okamoto S, Chinen H, Kobayashi T, Sato T, Sakuraba A, Kitazume MT, Sugita A, Koganei K, et al: Unique CD14 intestinal macrophages contribute to the pathogenesis of crohn disease via IL-23/IFN-gamma axis. J Clin Invest 118: 2269-2280, 2008.

26. Dalman MR, Deeter A, Nimishakavi G and Duan ZH: Fold change and p-value cutoffs significantly alter microarray interpretations. BMC Bioinformatics 13 (Suppl 2): S11, 2012.

27. Kim CH, Lillehoj HS, Hong YH, Keeler CL Jr and Lillehoj EP: Analysis of global transcriptional responses of chicken following primary and secondary eimeria acervulina infections. BMC Proc 5 (Suppl 4): S12, 2011.
28. Shi Q and Yang X: Circulating MicroRNA and long noncoding RNA as biomarkers of cardiovascular diseases. J Cell Physiol 231: 751-755, 2016.

29. Rahman SH, Ammori BJ, Holmfield J, Larvin $M$ and McMahon MJ: Intestinal hypoperfusion contributes to gut barrier failure in severe acute pancreatitis. J Gastrointest Surg 7: 26-36, 2003.

30. Zhang XP, Zhang J, Song QL and Chen HQ: Mechanism of acute pancreatitis complicated with injury of intestinal mucosa barrier. J Zhejiang Univ Sci B 8: 888-895, 2007.

31. Fillon S, Robinson ZD, Colgan SP and Furuta GT: Epithelial function in eosinophilic gastrointestinal diseases. Immunol Allergy Clin North Am 29: 171-178, xii-xiii, 2009.

32. Leveau P, Wang X, Sun Z, Börjesson A, Andersson E and Andersson R: Severity of pancreatitis-associated gut barrier dysfunction is reduced following treatment with the PAF inhibitor lexipafant. Biochem Pharmacol 69: 1325-1331, 2005.

33. Zhang JX, Dang SC, Yin K and Jiang DL: Protective effect of clodronate-containing liposomes on intestinal mucosal injury in rats with severe acute pancreatitis. Hepatobiliary Pancreat Dis Int 10: 544-551, 2011.

34. Yin K, Dang SC and Zhang JX: Relationship between expression of triggering receptor-1 on myeloid cells in intestinal tissue and intestinal barrier dysfunction in severe acute pancreatitis. World J Emerg Med 2: 216-221, 2011.

35. Carpenter S, Aiello D, Atianand MK, Ricci EP, Gandhi P, Hall LL, Byron M, Monks B, Henry-Bezy M, Lawrence JB, et al: A long noncoding RNA mediates both activation and repression of immune response genes. Science 341: 789-792, 2013.

36. Cui H, Xie N, Tan Z, Banerjee S, Thannickal VJ, Abraham E and Liu G: The human long noncoding RNA lnc-IL7R regulates the inflammatory response. Eur J Immunol 44: 2085-2095, 2014.

37. Liu R, Tang A, Wang X, Chen X, Zhao L, Xiao Z and Shen S: Inhibition of lncRNA NEAT1 suppresses the inflammatory response in IBD by modulating the intestinal epithelial barrier and by exosome-mediated polarization of macrophages. Int J Mol Med 42: 2903-2913, 2018.

38. Ma S, Ming Z, Gong AY, Wang Y, Chen X, Hu G, Zhou R, Shibata A, Swanson PC and Chen XM: A long noncoding RNA, lincRNA-Tnfaip3, acts as a coregulator of NF-kB to modulate inflammatory gene transcription in mouse macrophages. FASEB J 31: 1215-1225, 2017.

39. Zhao F, Qu Y, Liu J, Liu H, Zhang L, Feng Y, Wang H, Gan J, Lu R and Mu D: Microarray profiling and co-expression network analysis of LncRNAs and mRNAs in neonatal rats following hypoxic-ischemic brain damage. Sci Rep 5: 13850, 2015.

This work is licensed under a Creative Commons Attribution-NonCommercial-NoDerivatives 4.0 International (CC BY-NC-ND 4.0) License. 